\title{
Serotyping and Pathogenicity of Erysipelothrix Strains Isolated from Tonsils of Slaughter Pigs in Thailand
}

\author{
Toshio TAKAHASHI, Patanee SUNAMA ${ }^{1)}$, Jarunee SATRA ${ }^{1)}$, Nonglak CHOLSINDHU ${ }^{1)}$, Suneejit KONGTHON ${ }^{1)}$, \\ Wongkwan JITNUPONG ${ }^{2}$, Kinya YAMAMOTO, Mayumi KIJIMA and Susumu FURUUCHI ${ }^{3)}$
}

National Veterinary Assay Laboratory, Tokura 1-15-1, Kokubunji, Tokyo 185-8511, Japan, ${ }^{1)}$ Veterinary Biologics Assay Center, Pakchong Nakomrachasima, ${ }^{2}$ Department of Livestock Development, Prayathai, Bangkok 10400, Thailand, and ${ }^{3)}$ Japan International Cooperation Agency, Shibuya-ku, Yoyogi 2-1-1, Tokyo 151-8558, Japan

(Received 4 February 1999/Accepted 19 May 1999)

ABSTRACT. Erysipelothrix strains were isolated from the tonsils of 46 (15.0\%) of 307 apparently healthy slaughter pigs in Thailand during the period of August to September, 1997. A total of 27 of the 46 Erysipelothrix isolates could be classified into 5 serovars but the remaining 19 were untypable in this study. Of the 25 isolates serologically identified as Erysipelothrix rhusiopathiae, 20, 4, and 1 isolates belonged to serovars 2,12, and 17, respectively. Only 2 isolates from the tonsils belonged to Erysipelothrix tonsillarum and represented either serovar 7 or 10. Although the periods and the districts of the survey were limited, the information obtained in the present investigation demonstrates the presence of a variety of serovars in pigs in Thailand. Of 29 selected isolates belonging to serovars $2,7,10$, 12,17 , and untypable, only $5(17.2 \%)$ were virulent for both mice and pigs. Five of these virulent isolates belonging to serovars 2 and 12 killed less than $30 \%$ of mice immunized with a swine erysipelas bacterin commercially available in Thailand, suggesting that the vaccine elicited a sufficient immunity to these field isolates.—KEY wORDs: characterization, Erysipelothrix, slaughter swine, Thailand, tonsil. J. Vet. Med. Sci. 61(9): 1007-1011, 1999

Erysipelothrix rhusiopathiae causes swine erysipelas, resulting in enormous economic losses in swine-producing areas of the world. The clinical signs of swine erysipelas can be divided into three types: acute, subacute, and chronic. The organism also causes a variety of diseases in many species of wild and domestic mammals and birds, as well as erysipeloid, a skin disease of humans [22]. Despite its wide range of hosts and a large variety of serological characteristics, the genus Erysipelothrix was originally considered to consist of a single species biochemically and morphologically identified as E. rhusiopathiae. Recently, on the basis of DNA relatedness among E. rhusiopathiae strains, represented by all 23 serovars recognized, we reported that the genus Erysipelothrix contains two main genomic species, E. rhusiopathiae consisting serovars 1a, $1 \mathrm{~b}, 2,4,5,6,8,9,11,12,15,16,17,19$, and type $\mathrm{N}$ (type $\mathrm{N}$ strains do not produce any precipitating antibody against homologous and heterologous heat-stable extracts in rabbits), and Erysipelothrix tonsillarum including serovars $3,7,10,14,20,22$, and 23 [13].

Different tissues of apparently healthy pigs have been examined for the presence of E. rhusiopathiae by many researchers. The preponderance of isolations has been from the tonsils, although the organisms have been isolated from the intestinal tract, lymph nodes, gall bladder, joints, and bone marrow $[1,5,8,15]$. Thus, the tonsils are generally recognized as a site of localization by Erysipelothrix in swine.

In Thailand, the prevalence of swine erysipelas has become a problem with respect to animal hygiene and a commercial erysipelas bacterin, Erysorb ${ }^{\mathrm{R}}$ Plus (Hoechst), is widely used for prevention of the disease. However, there is a few information about isolation of Erysipelothrix from pigs and characterization of the isolates in Thailand [7]. The epidemiological features of the disease are still unclear. The primary objective of the present study was to investigate the serological characterization and pathogenicity of Erysipelothrix isolates from the tonsils of apparently healthy slaughter pigs. The secondary was to determine if the erysipelas bacterin commercially available in Thailand was effective to the field isolates.

\section{MATERIALS AND METHODS}

Tissues: Tonsils were obtained from 307 pigs, 80 to 100 $\mathrm{kg}$ in weight, selected at Pakchong Slaughterhouse, Nakomrachasima, and Ayutthaya Slaughterhouse, Ayutthaya, during the period of August to September 1997. These slaughterhouses were neighboring to the greatest pig production region in the center of Thailand. Tonsils from each pig were placed in vinyl specimen bags and transported in ice to the Veterinary Biologics Assay Center, Pakchong.

Culture procedure for isolation of Erysipelothrix: Approximately $1 \mathrm{~g}$ of tonsil tissue was diced into small pieces, and inoculated into $10 \mathrm{~m} l$ of tryptose phosphate (TP) broth (pH 7.6; Difco) containing 0.1\% Tween 80, $25 \mu \mathrm{g} / \mathrm{ml}$ gentamicin and $250 \mu \mathrm{g} / \mathrm{m} l$ kanamycin. After incubation at $37^{\circ} \mathrm{C}$ for $24 \mathrm{hr}$, one loopful of the culture was streaked onto each plates of TP agar ( $\mathrm{pH} 7.6$; Difco) containing $0.1 \%$ Tween $80,25 \mu \mathrm{g} / \mathrm{m} l$ gentamicin and $250 \mu \mathrm{g} / \mathrm{m} l$ kanamycin. The agar plates were incubated at $37^{\circ} \mathrm{C}$ for $24 \mathrm{hr}$ and then examined for the colony formation.

Identification of isolates: Representative colonies with typical morphologic characteristics of Erysipelothrix were 
picked from each plate, seeded into tubes of TP broth, and incubated at $37^{\circ} \mathrm{C}$ for $24 \mathrm{hr}$. The isolates were characterized by cellular morphology, typical reaction in triple sugar iron agar slants, "test-tube brush" growth in gelatin medium, and ability to clot the rabbit citrated plasma $[16,20]$ to choose candidates of Erysipelothrix species. The isolates were finally subjected to polymerase chain reaction (PCR) system [4] for identification of Erysipelothrix species.

Serotyping: The serovars of the isolates were determined by using an agar gel double-diffusion precipitation system as previously described $[2,17]$. Colonies from a 48-hr agar plate of each strain were inoculated into TP broth containing $0.1 \%$ Tween 80 , incubated at $37^{\circ} \mathrm{C}$ for $24 \mathrm{hr}$, and the culture was centrifuged at $12,000 \mathrm{~g}$ for $30 \mathrm{~min}$. The bacterial cells were washed three times with physiological saline and suspended in distilled water at 1/30 of the original volume. The bacterial suspension was autoclaved at $121^{\circ} \mathrm{C}$ for $1 \mathrm{hr}$, cooled, and clarified by centrifugation. The supernatant fluid was tested against typing sera (rabbit origin) representing all the known serovars, 1 through 23 and type $\mathrm{N}$, of the Erysipelothrix species.

Animals: A total of 2365 -week-old female mice of the ICR strain and 15 2-to 4-month-old female or castrated male Yorkshire pigs were used. They were conventionally farrowed and raised in confinement. Sera of the swine showed a growth-agglutination (GA) titer [9] $\leqq 8$.

Pathogenicity tests: Tests for pathogenic characteristics of 29 selected isolates were conducted as described previously $[18,25]$. Groups of 4 mice were inoculated subcutaneously with $0.1 \mathrm{~m} l$ of TP broth culture $\left(10^{8}\right.$ colony forming units $(\mathrm{CFU}) / \mathrm{m} l)$ of each isolate. The mortality of each mouse group was scored 14 days after inoculation.

One pig was inoculated intradermally on the right and left sides of the flank with $0.1 \mathrm{ml}\left(10^{8} \mathrm{CFU}\right)$ of TP broth of six different isolates (six inocula per pig); three isolates were injected separately in a horizontal row on one side of the flank. Clinical signs of these pigs were examined every day for 14 days after inoculation. The strain that induced a local urticarial lesion $\geqq 20 \mathrm{~mm}$ in diameter in 14 days after inoculation was assigned to be virulent for pigs.

Protection studies: An imported current erysipelas bacterin (serovars 1 and 2), Erysorb ${ }^{\mathrm{R}}$ Plus (Hoechst), conferring $100 \%$ protection upon mice against challenge with 100LD 50 of E. rhusiopathiae type strain (ATCC19414, serovar 2), was used for the protection test. Five field isolates of E. rhusiopathiae belonging to either serovar 2 or 12 were used for challenge. Mice were immunized subcutaneously in the right inner thigh with $0.5 \mathrm{ml}$ of the vaccine, and boosted once with the same dose 14 days later. Fourteen days after boosting, the mice were allotted into groups of ten and challenged subcutaneously in the left inner thigh with $100 \mathrm{LD}_{50}$ of the 5 field isolates. The same number of non-immunized mice were used for control. The mice were observed for death for a period of 14 days.

Pigs were immunized subcutaneously at the cervical area just caudal to the ear with $2 \mathrm{~m} l$ of the vaccine, and boosted once with the same dose 14 days later. Fourteen days after boosting, groups of two pigs consisting one vaccinated pig and a non-vaccinated pig were challenged intradermally in the flank with $10^{7} \mathrm{CFU}$ of field isolates and were observed for clinical signs for 14 days. For determination of the GA titer of porcine sera, blood samples were collected from the cranial vena cava of pigs at challenge.

\section{RESULTS}

Bacterial isolation and serotyping: Samples of the tonsils collected from 307 slaughter pigs were surveyed by bacteriological and molecular genetical procedures described in the Materials and Methods. The results of serotyping were summarized in Table 1. Erysipelothrix species could be isolated and identified from $46(15.0 \%)$ samples. One representative isolate from each sample was chosen for further study. Among them, 27 isolates could be serotyped: 25 isolates were E. rhusiopathiae serovars and 2 were $E$. tonsillarum serovars. The remaining 19 were untypable in this study. Among 25 isolates classified into $E$. rhusiopathiae serovars, serovar 2 was predominant (80.0\%). Patterns of the frequency of isolation of the serovars varied among the 11 sampling days (data not shown).

Pathogenicity of isolates: Results of tests for pathogenic characteristics of selected isolates are shown in Table 2. Of 19 isolates belonging to serovar 2, $17(89.5 \%)$ were avirulent for mice and pigs. Although all of the mice-virulent isolates of serovar 12 induced local urticarial lesions at the site of inoculation in pigs, the 2 untypable mice-virulent isolates

Table 1. Serovars of Erysipelothrix isolates from tonsils of slaughter pigs in Thailand

\begin{tabular}{|c|c|c|c|c|c|c|}
\hline \multirow[t]{2}{*}{ Place } & \multicolumn{3}{|c|}{$\begin{array}{l}\text { E. rhusiopathiae } \\
\text { serovars }\end{array}$} & \multicolumn{2}{|c|}{$\begin{array}{l}\text { E. tonsillarum } \\
\text { serovars }\end{array}$} & \multirow[t]{2}{*}{ Untypable $^{a)}$} \\
\hline & 2 & 12 & 17 & 7 & 10 & \\
\hline Pakchong & 3 & 1 & 1 & 1 & 1 & 10 \\
\hline Ayutthaya & 17 & 3 & & & & 9 \\
\hline Total & 20 & 4 & 1 & 1 & 1 & 19 \\
\hline
\end{tabular}

a) Serovars could not be determined with typing sera representing known serovars. 
Table 2. Pathogenicity of Erysipelothrix strains for mice and pigs

\begin{tabular}{cccccc}
\hline Species serovar & $\begin{array}{l}\text { No. of strains } \\
\text { examined }\end{array}$ & \begin{tabular}{l} 
Pathogenicity for mice \\
\cline { 3 - 6 }
\end{tabular} & Virulent & Avirulent & Pathogenicity for pigs ${ }^{\mathrm{b})}$ \\
\hline E. rhusiopathiae & 19 & 2 & 17 & 2 & Avirulent \\
2 & 4 & 3 & 1 & 3 & 17 \\
12 & 1 & 0 & 1 & 0 & 1 \\
17 & 1 & 0 & 1 & 0 & 1 \\
E. tonsillarum & 1 & 0 & 1 & 0 & 1 \\
7 & 3 & 2 & 1 & 0 & 3 \\
10 & 29 & 7 & 22 & 5 & 24 \\
Untypable & & & & & \\
\hline Total & & &
\end{tabular}

a) Virulent; mice dead within 14 days after subcutaneous injection of $10^{8}$ viable bacteria. Avirulent; mice not visibly affected by injection.

b) Virulent; acute local urticarial lesions $\geqq 20 \mathrm{~mm}$ in diameter in pigs after intradermal injection of $10^{8}$ viable bacteria. Avirulent; lesions were not induced in pigs.

Table 3. Protection of mice against virulent E. rhusiopathiae isolates by vaccination with the Thai commercial erysipelas bacterin

\begin{tabular}{lcc}
\hline \multirow{2}{*}{$\begin{array}{c}\text { Challenge } \\
\text { strain (serovar) }\end{array}$} & \multicolumn{2}{c}{ N. of survived mice } \\
\cline { 2 - 3 } & Vaccinated group & Non-vaccinated group \\
\hline PT-56 (2) & 9 & 0 \\
AT3-36 (2) & 10 & 0 \\
PT-5 (12) & 7 & 0 \\
AT2-8 (12) & 7 & 0 \\
AT2-13 (12) & 7 & 0 \\
\hline
\end{tabular}

a) Mice were immunized with vaccine and boosted 14 days later. Fourteen days after boosting, ten mice were challenged subcutaneously with $100 \mathrm{LD}_{50}$ of virulent field isolates of serovars 2 and 12 . Survival of mice were scored 14 days after challenge.

did not induce any clinical signs in pigs. In total, only 5 $(17.2 \%)$ of 29 selected isolates belonging to serovars 2,7 , $10,12,17$, and untypable were virulent for both mice and pigs.

Protection studies: Mice immunized subcutaneously with the commercial vaccine were challenged with $100 \mathrm{LD}_{50}$ of the 5 virulent field isolates 14 days post immunization. More than $70 \%$ of vaccinated mice survived after challenge with all the isolates, whereas all control mice died within 3 to 5 days after challenge (Table 3 ).

Pigs immunized subcutaneously with the above vaccine were challenged with the 5 virulent isolates 14 days after boosting. None of vaccinated pigs showed any clinical signs of acute erysipelas (Table 4). All control pigs showed local urticarial lesions at the site of intradermal inoculation. The GA titers of the vaccinated pigs were $\geqq 32$, whereas nonvaccinated pigs retained at a GA titer of 4 .

\section{DISCUSSION}

It is well documented that the prevalence of $E$.
Table 4. Clinical responses of pigs vaccinated with the Thai commercial erysipelas bacterin against challenge with the virulent isolates of E. rhusiopathiae

\begin{tabular}{lccc}
\hline $\begin{array}{l}\text { Challenge } \\
\text { strain }^{\text {a) }} \text { (serovar) }\end{array}$ & Vaccination $^{\text {b) }}$ & GA titer $^{\text {c }}$ & Responses $^{\mathrm{d})}$ \\
\hline PT-56 (2) & + & 32 & - \\
PT-56 (2) & - & 4 & $12 \times 10$ \\
AT3-36 (2) & + & 64 & - \\
AT3-36 (2) & - & 4 & $7 \times 5$ \\
PT-5 (12) & + & 64 & - \\
PT-5 (12) & - & 4 & $17 \times 15$ \\
AT2-8 (12) & + & 64 & - \\
AT2-8 (12) & - & 4 & $15 \times 15$ \\
AT2-13 (12) & + & 64 & - \\
AT2-13 (12) & - & 4 & $10 \times 8$ \\
\hline
\end{tabular}

a) Pigs were immunized with the commercial vaccine and boosted 14 days later. Fourteen days after boosting, pigs were challenged with $10^{8} \mathrm{CFU}$ of virulent isolates of serovars 2 and 12 .

b) +: Vaccinated, -: Non-vaccinated.

c) Growth-agglutination titer. Sera of pigs were collected on the day of challenge.

d) Fourteen days after challenge, pigs were examined for erythema. Erythema were expressed as the major axis $(\mathrm{cm}) \times$ the minor axis $(\mathrm{cm})$ appeared on their skin at the site of challenge.

rhusiopathiae among porcine carriers examined ranges from 3 to $98 \%$, with most surveys indicating that 20 to $40 \%$ of pigs are carriers $[5,11,15,18,19]$. In this study, Erysipelothrix was isolated from tonsils of 46 (15.0\%) of 307 apparently healthy slaughter pigs in Thailand. The results suggested that the relatively high tonsillar carrier rate was associated with occurrence of swine erysipelas. Therefore, as in other swine-producing areas of the world, the organism is apparently ubiquitous in swine and in the environment as a soil contaminant [25], and probably constitutes a significant potential pathogen for pigs in Thailand.

Pathanasophon et al. [7] have reported the results of 
serotyping of Erysipelothrix strains isolated from the tonsils of apparently healthy slaughter pigs (116 isolates) and the lesions of diseased pigs (14 isolates) during a period from 1980 to 1987 in Thailand. The majority of the 22 recognized serovars were found in these isolates from porcine tonsils: serovars $2,6,9$, and 5 were the most prevalent $(33.6 \%$, $12.9 \%, 6.9 \%$, and $5.2 \%$, respectively). All of the clinical isolates belonged to serovar 1a. However, pathogenicity of the field isolates in Thailand has not yet been characterized. The present investigation also demonstrated the presence of a variety of serovars of Erysipelothrix, even though it was undertaken in the comparatively limited periods and districts. Similar results on the wide distribution of serovars have been reported in the other countries by Wood and Harrington [24] and Kucsera [3]. Serovar 2 of E. rhusiopathiae was also found to be the most prevalent $(43.5 \%)$ in this study, and the remaining 4 serovars typable in this study shared $15.2 \%$ of the total isolates. The predominant serovars of isolates in Thailand were moderately consistent with those reported previously [3, 14, $15,17,18,24]$. The serovars of 19 isolates $(41.3 \%)$ were untypable. In Thailand, there have been limited attempts to serotype Erysipelothrix isolates. It is therefore possible that these untypable isolates consist of 1 or more unknown serovars unclassified so far or newly established serovars 24, 25, and 26 [6]. According to results on the PCR amplification system using the DNA sequence coding $16 \mathrm{~S}$ rRNA, the untypable isolates could also be genetically identified as Erysipelothrix species. Further studies are needed on the serotyping of the untypable isolates by testing heat-extracted antigen of serovar reference strains with antisera prepared in rabbits against the untypable isolates.

Previously, we reported that a cluster of avirulent strains of serovar 7 existing predominantly in tonsils of apparently healthy pigs in Japan was genetically distinct from the majority of E. rhusiopathiae strains. These DNA-DNA hybridization results established the transfer of these serovar 7 organisms to a new species, Erysipelothrix tonsillarum [12]. However, results of present investigation did not show such predominance of E. tonsillarum in Thailand. The distribution may vary among countries or regions surveyed in each country.

E. rhusiopathiae of serovars 1 and 2 have been generally believed to be the predominant causative agents of swine erysipelas $[14,21]$. Half of the total isolates from porcine tonsils in the present survey belonged to serovar 2. Most of the isolates belonging to serovar 2 were characterized by the lack of pathogenicity for mice and pigs. The virulence factor of E. rhusiopathiae has not yet been clearly understood. Present results on the pathogenicity test demonstrate drastic differences in pathogenic characteristics among the E. rhusiopathiae isolates of serovar 2, indicating that these strains may constitute an excellent tool to study the pathogenesis. The reasons for such occurrence of avirulent isolates in serovar 2 could not be explained from the epidemiological point of view. In any case, some isolates of E. rhusiopathiae serovars 2 and 12 were virulent for pigs, capable of inducing urticarial lesions at the site of intradermal challenge. It is likely that the virulent $E$. rhusiopathiae in tonsils may represent not only a route of primary infection but also a potential source of infection. In contrast, the avirulent strains such as E. tonsillarum would be residents in porcine tonsils.

Wood et al. [23] reported that some isolates of $E$. rhusiopathiae of serovars 9 and 10 caused acute local or generalized urticarial lesions in pigs and caused fatal septicemia in mice vaccinated with standard erysipelas adsorbate bacterins made from strains of serovar 2 . In the present experiments, the commercial bacterin (serovars 1 and 2) in Thailand conferred upon pigs an immunity from the challenge of all the isolates tested. Vaccinated mice $(30 \%)$ died after heterologous challenge exposure with the isolates of serovar 12, although nearly complete protection was induced in vaccinated mice against the isolates of serovar 2. This observation may be attributed to a higher susceptibility of mice to the challenge strains of $E$. rhusiopathiae than that of pigs. Presumably, the induction of death in vaccinated mice by challenge isolates suggests that the immunological characteristics are distinct from those of the strains used in the preparation of the erysipelas bacterin. Sawada and Takahashi [10] reported that differences in protection levels of mice and pigs immunized with erysipelas vaccine composed of culture filtrate against challenge exposure with strains of various serovars were caused by quantitative (rather than qualitative) antigenic dissimilarility between the vaccine strain and certain challenge strains. Therefore, further investigation with the cross protection experiment using the prevalent clinical isolates of some serovars in swine is needed to evaluate the efficacy of the erysipelas bacterin in the field of Thailand.

\section{REFERENCES}

1. Connel, R. and Langford, E.V. 1953. Studies of swine erysipelas. V. Presence of Erysipelothrix rhusiopathiae in apparently healthy slaughter pigs. Can. J. Comp. Med. 17: 448-453.

2. Kucsera, G. 1973. Proposal for standardization of the designations used for serotypes of Erysipelothrix rhusiopathiae (Migula) Buchanan. Int. J. Syst. Bacteriol. 23: 184-188.

3. Kucsera, G. 1979. Serological typing of Erysipelothrix rhusiopathiae and epidemiological significance of the typing. Acta Vet. Acad. Sci. Hung. 22: 251-261.

4. Makino, S., Okada, Y., Maruyama, T., Ishikawa, K., Takahashi, T., Nakamura, M., Ezaki, T. and Morita, H. 1994. Direct and rapid detection of Erysipelothrix rhusiopathiae DNA in animals by PCR. J. Clin. Microbiol. 32: 1526-1531.

5. Murase, N. and Ebi, Y. 1960. Studies on the typing of Erysipelothrix rhusiopathiae. IV. Epidemiological significance of Erysipelothrix rhusiopathiae harbored in the tonsils of apparently healthy pigs. Jpn. J. Vet. Sci. 21: 1-10.

6. Nørrung, V. and Molin, G. 1991. A new serotype of Erysipelothrix rhusiopathiae isolated from pig slurry. Acta Vet. Hung. 39: 137-138.

7. Pathanasophon, P., Tanticharoenyos, T. and Pramoolsinsap. 1990. Serotypes of Erysipelothrix rhusiopathiae isolated from 
swine in Thailand. J. Thai Vet. Med. Assoc. 41: 101-106 (In Thai).

8. Rowsell, H.C. 1958. A culture and biochemical study of strains of Erysipelothrix rhusiopathiae with special reference to the carrier pig. Can. J. Comp. Med. 22: 82-86.

9. Sawada, T., Muramatu, M. and Seto, K. 1979. Responses of growth agglutinating antibody and protection of pigs inoculated with swine erysipelas live vaccine. Jpn. J. Vet. Sci. 41: 593-600.

10. Sawada, T. and Takahashi, T. 1987. Cross protection of mice and swine inoculated with culture filtrate of attenuated Erysipelothrix rhusiopathiae and challenge exposed to strains of various serovars. Am. J. Vet. Res. 48: 239-242.

11. Stephenson, E.H. and Berman, D.T. 1978. Isolation of Erysipelothrix rhusiopathiae from soil and manure of swine-raising premises. Am. J. Vet. Res. 39: 187-188.

12. Takahashi, T., Fujisawa, T., Benno, Y., Tamura, Y., Sawada, T., Suzuki, S., Muramatsu, M. and Mitsuoka, T. 1987. Erysipelothrix tonsillarum sp. nov. isolated from tonsils of apparently healthy pigs. Int. J. Syst. Bacteriol. 37: 166-168.

13. Takahashi, T., Fujisawa, T., Tamura, Y., Suzuki, S., Muramatsu, M., Sawada, T., Benno, Y. and Mitsuoka, T. 1992. DNA relatedness among Erysipelothrix rhusiopathiae strains representing all twenty-three serovars and Erysipelothrix tonsillarum. Int. J. Syst. Bacteriol. 42: 469-473.

14. Takahashi, T., Nagamine, N., Kijima, M., Suzuki, S., Takagi, M., Tamura, Y., Nakamura, M., Muramatsu, M. and Sawada, T., 1996. Serovars of Erysipelothrix strains isolated from pigs affected with erysipelas in Japan. J. Vet. Med. Sci. 58: 587589.

15. Takahashi, T., Sawada, T., Muramatsu, M., Tamura, Y., Fujisawa, T., Benno, Y. and Mitsuoka, T. 1987. Serotypes, antimicrobial susceptibility and pathogenicity of Erysipelothrix rhusiopathiae isolates from tonsils of apparently healthy slaughter pigs. J. Clin. Microbiol. 25: 536-539.
16. Takahashi, T., Takahashi, I., Tamura, Y., Sawada, T., Yoshida, T., Suzuki, S. and Muramatsu, M. 1990. Mechanism of plasma clotting by Erysipelothrix rhusiopathiae. J. Clin. Microbiol. 28: 2161-2164.

17. Takahashi, T., Sawada, T., Takagi, M., Seto, K., Kanzaki, M and Maruyama, T. 1984. Serotypes of Erysipelothrix rhusiopathiae strains isolated from slaughter pigs affected with chronic erysipelas. Jpn. J. Vet. Sci. 46: 149-153.

18. Takahashi, T., Zarkasie, K., Mariana, S., Sumadi and Ogata, M. 1989. Serological and pathogenic characterization of Erysipelothrix rhusiopathiae isolates from tonsils of slaughter pigs in Indonesia. Vet. Microbiol. 21: 165-175.

19. Timoney, J. 1970. Seasonal variations in the tonsillar carrier rate of Erysipelothrix rhusiopathiae in Irish market pigs. Irish Vet. J. 24: 81-85.

20. Wood, R.L. 1970. Erysipelothrix. pp. 101-105. In: Manual of Clinical Microbiology (Blair, J.E., Lennette, E.H. and Truant, J.P. eds.). American Society for Microbiology, Washington, D.C.

21. Wood, R.L. 1984. Swine erysipelas - a review of prevalence and research. J. Am. Vet. Med. Assoc. 184: 944-948.

22. Wood, R.L. 1993. Swine erysipelas. pp. 475-486. In: Disease of Swine, 7th ed. (Leman, A.D., Straw, B.E., Mengeling, W.L., Allarie, S.D. and Taylor, D.J. eds.). Iowa State University Press, Ames.

23. Wood, R.L., Booth, G.D. and Cutlip, P.C. 1981. Susceptibility of vaccinated swine and mice to generalized infection with specific serotypes of Erysipelothrix rhusiopathiae. Am. J. Vet. Res. 42: 608-614.

24. Wood, R.L. and Harrington, R., Jr. 1978. Serotypes of Erysipelothrix rhusiopathiae isolated from swine pens in the United States. Am. J. Vet. Res. 39: 1833-1840.

25. Wood, R.L. and Packer, R.A. 1972. Isolation of Erysipelothrix rhusiopathiae from soil and manure of swine-raising premises. Am. J. Vet. Res. 33: 1611-1620 